Bulletin of the Natural History Museum, 2016, 7: 31-66.

Received 14 Apr 2016; Accepted 29 Nov 2016.

doi:10.5937/hnhmb1609031S

UDC: $582.282 .14(497.113)$

Original scientific paper

\title{
LEOTIOMYCETES CHECKLIST OF MT FRUŠKA GORA WITH NEW RECORDS FOR SERBIAN MYCOBIOTA
}

\author{
DRAGIŠA SAVIĆ ${ }^{1}$, MAJA KARAMAN ${ }^{2}$ \\ ${ }^{1}$ National Park Fruška Gora, Zmajev trg 1, 21208 Sremska Kamenica, Republic of \\ Serbia, e-mail: dragita@gmail.com \\ ${ }^{2}$ University of Novi Sad, Faculty of Sciences, Department of Biology and \\ Ecology, Dositeja Obradovića Square 2, 21000 Novi Sad, Republic of Serbia
}

In the period from 2012 to 2016 diversity of fungi from the class Leotiomycetes (Ascomycota) was investigated on the Mt Fruška Gora Mountain (Serbia). In total we recorded 164 species of which 131 represent the first records for Serbia. The paper includes a list of all the collected leotiomycetous fungi, except order Erysiphales, on Mt Fruška Gora, with data of localities, collection dates, host plants, as well as data from the literature concerning all species that have so far been reported from Serbia. The whole number of recorded species from the class Leotiomycetes in Serbia is 342, including the species from the order Erysiphales (104).

Key words: Ascomycota, Leotiomycetes, Serbia

\section{INTRODUCTION}

Fruška Gora Mountain is located between $45^{\circ} 0^{\prime}$ and $45^{\circ} 15^{\prime} \mathrm{N}$ and between $16^{\circ} 37^{\prime}$ and $18^{\circ} 01^{\prime}$ E. It extends between the Danube and Sava rivers (Miljković 1975). This mountain, located in the south of the Pan- 
nonian Plain, has a length of about $80 \mathrm{~km}$, and a maximum width of $15 \mathrm{~km}$, with the highest peak, Crveni Čot, at $539 \mathrm{~m}$. Hydrographic network of Mt Fruška Gora is very rather and relatively evenly distributed. Surface water is represented with a network of streams, ponds and artificial lakes. Fruška Gora is located at the border of the temperate continental and subcontinental climate region, and due to the changes in climatic characteristics along altitudinal gradients and the influence of forest cover, the climate here has subcontinental characteristics (Stevanović and Stevanović 1995). According to the biogeographical classification of the territory of Serbia, Fruška Gora belongs to the Central European region and within it to the Central European Balkan-subregion of the Illyrian or Pannonian province (Stevanović 1995). Fruška Gora is a forest area, but from the former 130,000 ha of land under forests, only 23,000 ha remain today, mainly because of anthropogenic factors and excessive exploitation in the past. At present, the former forest areas are replaced mostly by meadows, fields, vineyards and orchards. Forest communities are of mixed or pure composition. Uniform forests are made of oak (Quercus petraea), beech (Fagus sylvatica) and sometimes linden (Tilia tomentosa) or hornbeam (Carpinus betulus), while the structure of mixed forests consists mostly of linden and beech, and rarely hornbeam and oak. Pure beech and pure hornbeam forests are rare while meadow-steppes are situated mainly on the periphery of the mountain (Janković \& Mišić 1980).

Serbia is mycologically a poorly explored area and most of the data is mainly related to species of the phylum Basidiomycota. The first data of the fungi of the phylum Ascomycota in Serbia was given by Schulzer et al. (1866). In his work about the plants of Slavonia (today in Republic of Croatia), data on nearly 1,000 species of fungi is presented, including around 140 species from the area of Fruška Gora (mainly Basidiomycota). In this work, Coccomyces dentatus and Ascodichaena rugosa recorded on Fruška Gora virtually represent the first published data about fungi of class Leotiomycetes in Serbia. After this work Nikola Ranojević published his data in two extensive articles in 1910 and 1914. Among more than 600 species that have been recorded in Serbia, most of them belong to phylum Ascomycota, and among them 30 species are members of the class Leotiomycetes. Until the end of the XX century, after his work, there were only three published papers on this group of fungi: Čolić (1967) reported 9 species for the area of the mountain Tara (Chlorociboria aeruginosa, Phaeohelotium fagineum, Mitrula paludosa, Cudonia circinans, Lirula macrospora, Lirula nervisequa, Rhytisma acerinum, $R$. salicinum and $R$. umbonatum). Gajić (1989) reported only 8 species for the Mountain Golija (Cenangium ferruginosum, Gremmenia infestans, Cyclaneusma minus, Cyclaneusma niveum, Lirula macrospora, Lirula nervisequa, Lophodermium pinastri i Lophodermium seditiosum) and Čoja (1989) only one. Since 
the beginning of the XXI century, more and more data of ascomycetous fungi of Serbia was obtained, thanks to the research of mushrooms in the forest ecosystems of Serbia (e.g. Karadžić 2003, 2006, 2008, Karadžic \& Milijašević 2003, 2005, 2008, Karadžić \& Stanivuković 2010). Since the most complete work regarding the ascomycetous fungi is present in the monograph about the order Erysiphales in Serbia (Ranković 2002) which includes data abot 104 species from this order, in this paper these data are not included.

The published data on fungi of class Leotiomycetes on Fruška Gora are extremely scarce. After first record obtained by Schulzer et al. (1866), Ranković (1955) cites Rhytisma acerinum, Karadžić (2011) reports Encoelia glaberrima, Pezicula carpinea and Rutstroemia bolaris, and Marković (2012) Phacidium lauri and Botryotinia fuckeliana. In the paper of Karaman et al. (2012) preliminary data based on studies ascomycetous fungi of Fruška Gora Mt. in the last 20 years is presented. Of the approximately 100 species that are reported in this paper, 20 belong to the class Leotiomycetes (Arachnopeziza aurata, Chlorociboria aeruginascens, Drepanopeziza punctiformis, etc.).

In the kingdom of fungi, the class Leotiomycetes belongs to the phylum Ascomycota and subphylum Pezizomycotina. In previous works (e.g. Dennis 1978, Kimbrough 1970, Pfister \& Kimbrough 2001) classification within this class was mostly based on morphological characters while molecular phylogenetic analysis of this group of fungi, that is comparatively young, brings some new facts in classification (Gernandt et al. 2001, Wang et al. 2006a). According to Wang et al. (2006b), the class Leotiomycetes was divided into 5 orders (Cyttariales, Erysiphales, Helotiales, Rhytismatales, Thelebolales). Subsequently, the orders Leotiales, Phacidiales and Thelocarpales were added (Crous et al. 2014, Jaklitsch et al. 2016). The largest order in the class Leotiomycetes (and in general Ascomycota) is the order Helotiales with about 2,500 species described so far (Lumbsch \& Huhndorf 2010).

The Leotiomycetes is a highly diverse group of Pezizomycotina and includes nonlichenized fungi producing a generally small apothecia with an exposed hymenium and an inoperculate, unitunicate ascus that has an apical perforation pore for releasing ascospores (Wang et al. 2006b).

In many cases the ecological and biological features of these fungi represent the important taxonomic characters. In some orders, such as the Erysiphales, Cyttariales, Thelebolales and Rhytismatales, distinct ecological characters and nutritional modes are almost unique for each group. Members of the Helotiales thrive in various ecosystems and cover a broad range of niches, thus helotialean fungi have been described as plant pathogens, endophytes, mycorrhizae, ectomycorrhizal parasites, fungal pa- 
rasites, terrestrial saprobes, aquatic saprobes, root symbionts and wood-rot fungi (Wang et al. 2006a).

\section{MATERIALS AND METHODS}

Samples of fungi were collected in the period from 2012 to 2016 on Mt. Fruška Gora (Serbia). Fungi were collected in different habitats, mostly in deciduous forests (localities: Iriški Venac, Novo Hopovo, Paragovo, Popovica, Čerević, Papratski Do, Andrevlje, Rakovac, Stražilovo, Ledinci, Vrdnik, Irig, Jabuka, Kraljeva stolica, Rajkovac, Testera, Osovlje and Zmajevac), on the meadow-steppe (localities: Glavica, Brankovac, Banstol, Bukovac, Erdelj and Grgurevci) and humid places near the streams and the Danube river (localities: Beočin, Sremska Kamenica, Sviloš, Sremski Karlovci, Susek, Banoštor and Petrovaradin).

Microscopic features of most samples were examined in the fresh, living state in tap water (Baral 1992) using a transmission light microscope (Olympus BX 51) with bright-field technique. Freehand sections were made using a razor blade. The iodine reaction was tested with Lugol's solution with a concentration of iodine around $0.5-1 \%$ (Baral 1987). In the case of herbarium specimens, hymenial fragments were rehydrated with water.

Identification of the species was performed using specific identification keys (e.g. Dennis 1978, Ellis \& Ellis 1997, Medardi 2012). The collected samples were dried and deposited in the officially unregistered Herbarium of Fruška Gora National Park (listed species designation F.G.). Author citations are given according to Index Fungorum (2016). Taxonomy and nomenclature (families and genera) are given according to Baral et al. (2015) and Jaklitsch et al. (2016). Taxa within families are listed in alphabetical order. For the species Leptodontidium trabinellum, Monostichella salicis, Polydesmia pruinosa and Rhynchosporium secalis affiliation to a certain family is still not determined, therefore they are in the group marked as "incertae sedis". Species of the genera Durella and Strossmayeria, which until now belonged to the family Helotiaceae (Kirk et al. 2008), are temporarily placed in a group named as "Strossmayeria lineage" according to Jaklitsch et al. (2016).

The following information is provided for each fungal species: scientific name, locality, date, host, herbarium number, collector and a person who identified given collection (leg. \& det.). For the reason of transparency, localities which refer to Fruška Gora were separated from the rest of Serbia. Only findings from Fruška Gora are enumerated in the list of species. Species which represent the first records for Serbia are marked 
with an asterisk. Species found in the anamorph state (asexual reproductive stage) are indicated as "(anamorph)", and species, for which a sexual reproductive stage (teleomorph) is unknown, e.g. Botrytis galanthina, have the notification "(Anamorphic Botryotinia)" after the name.

\section{RESULTS AND DISCUSSION}

Based on our research results obtained in this study for the Fruška Gora Mountain and previous literature data for the whole region of Serbia (Schulzer et al. 1866, Ranojević 1910, 1914, Ranković 1955, 2002, Čolić 1967, Gajić 1989, Čoja 1989, Szabados 1998, Karadžić 2003, Karadžić et al. 2002, 2004, Milijašević 2003, Karadžić \& Milijašević 2003, 2005, 2008, Milijašević \& Karadžić 2004, Bulaić et al. 2006, Keča 2006, Marković 2006, Marković \& Karadžić 2006, Miletić \& Stević 2006, Ivančević 2007, Karadžić \& Milanović 2008, Halaši et al. 2008, Karadžić \& Stanivuković 2010, Ivančević \& Davidović 2011, Karaman et al. 2012, Marković 2012, Golubović-Ćurguz et al. 2013 and Hrustić et al. 2015) the total number of known fungal species from the class Leotiomycetes is 342 species up to date. About one third of them belong to the order Erysiphales (104) and the rest to the orders Helotiales (196), Rhytismatales (30), Phacidiales (9) and Thelebolales (3).

The total number of species of Leotiomycetes on Fruška Gora is 180, and 148 of them represent new records for the studied area, and 131 the first records for Serbia.

Most of the recorded species on Fruška Gora were found in the teleomorphic state, with four species found in both teleomorphic and anamorphic state (Ascocoryne sarcoides, Holwaya mucida, Sclerotinia sclerotiorum and Dermea prunastri) and only eight in the anamorphic state (Ascocoryne solitaria, Botryotinia fuckeliana, Botrytis galanthina, Drepanopeziza punctiformis, Drepanopeziza sphaerioides, Monilinia fructigena, Sclerotinia sclerotiorum and Symphyosirinia clematidis).

According to the type of substrate, most species on Fruška Gora grow as saprotrophs on rotten wood and fallen branches of deciduous trees (78), on rotting leaves of deciduous trees (19), on rotting leaves and cones of conifers (10), on catkins of alder and hazel (2), on cupules and fruits (oak, beech, alder) (21) and on rotten herbaceous plants (39). In addition, one species (Dumontinia tuberosa) is a parasite on the plant rhizomes of Anemone ranunculoides and one (Polydesmia pruinosa) occurs primarily on stromata of pyrenomycetes. Rare substrates for this group of fungi are soil, on which Leotia lubrica and Geoglossum fallax occur (terricolous) and 
dung of mammals, where three coprophilous species was detected (Thelebolus stercoreus, Trichobolus sphaerosporus and T. zukalii)

We have made corrections on two misidentified collections previously published from Mt. F.G. (Karaman et al. 2012) on the basis of reexamination of dried material. These are: F.G. 54, found in Beočin on the leaf of white willow and identified as Marssonina salicina Tehon that in fact represents Drepanopeziza sphaerioides (Pers.) Nannf., and F.G. 87, found at the site of Kraljeva stolica and identified as Lachnellula subtilissima (Cooke) Dennis, that in fact represents the immature unknown fungus from division Basidiomycota.

\section{Species List}

Class LEOTIOMYCETES O.E. Erikss. \& Winka 1997

Ordo: Helotiales Nannf. 1932

Fam.: Arachnopezizaceae Hosoya, J.G. Han \& Baral 2015

1. Arachnopeziza aurata Fuckel

Fruška Gora: Iriški Venac, Karaman et al. 2012: 43; Andrevlje, 21.10.2013., on Tilia tomentosa, dead wood, F.G. 408, leg. \& det. D. Savić.

2. Arachnopeziza aurelia (Pers.) Fuckel

Fruška Gora: Zmajevac, 30.03.2016., on Quercus petraea, cupula, F.G. 405, leg. \& det. D. Savić.

Serbia: northeastern part, Karadžić \& Milijašević 2005: 73, on Quercus petraea.

3.*Arachnoscypha aranea (De Not.) Boud.

Fruška Gora: Papratski Do, 27.10.2015., on Quercus petraea, cupula, F.G. 406, leg. \& det. D. Savić.

4.*Eriopezia caesia (Pers.) Rehm

Fruška Gora: Iriški Venac, 28.02.2012., on Tilia tomentosa, dead wood, F.G. 407, leg. \& det. D. Savić.

Fam.: Ascodichaenaceae D.Hawksw. \& Sherwood 1982

5. Ascodichaena rugosa Butin

Fruška Gora: Schulzer et al. 1866: 40, sub Radulum fagineum.

Serbia: Mt Avala, Ranojević 1910: 376, sub Radulum fagineum, on Carpinus betulus and Fagus sylvatica; more locations, Karadžić 2003: 61, on Fagus sylvatica; Karadžić \& Milijašević 2003: 27, on F. sylvatica; northeastern part, Karadžić \& Milijašević 2005: 73, on Quercus petraea; Karadžić 2012: 3, on F. sylvatica. 
Fam.: Calloriaceae Marchand 1894

6.*Calloria neglecta (Lib.) B.Hein

Fruška Gora: Paragovo, 03.04.2013., on Urtica dioica, dead stem, F.G. 466, leg. \& det. D. Savić.

7.*Duebenia compta (Sacc.) Nannf. ex B. Hein

Fruška Gora: Glavica, 25.03.2014., on Lathyrus latifolius, dead stem, F.G. 334, leg. \& det. D. Savić.

Fam.: Cenangiaceae Rehm 1888

Cenangium acuum Cooke \& Peck

Serbia: Milijašević \& Karadžić 2004: 122, on Pinus nigra and Pinus sylvestris; Karadžić \& Stanivuković 2010: 3, on P. nigra and $P$. sylvestris; more locations, Karadžić \& Milijašević 2008: 150, on $P$. nigra and $P$. sylvestris.

8. ${ }^{*}$ Cenangium alnicola Hazsl.

Fruška Gora: Ledinci, 01.11.2016., on Fagus sylvatica, branch, F.G. 839, leg. \& det. D. Savić.

Cenangium ferruginosum Fr.

Serbia: Karadžić \& Stanivuković 2010: 3, on Pinus nigra and Pinus sylvestris; Mt Golija (Popaljenik), Gajić 1989: 61, on $P$. nigra; more locations, Karadžić \& Milijašević 2008: 150, on $P$. nigra and $P$. sylvestris.

9.*Encoelia furfuracea (Roth) P.Karst.

Fruška Gora: Paragovo, 07.02.2014., on Carpinus betulus, branch, F.G. 571, leg. \& det. D. Savić; Andrevlje, 26.02.2014., on Alnus glutinosa, branch, F.G. 409, leg. \& det. D. Savić.

10. Encoelia glaberrima (Rehm) Kirschst.

Fruška Gora: Karadžić 2011: 3, on Carpinus betulus; Andrevlje, 20.11.2012., on C. betulus, branch, F.G. 572, leg. \& det. D. Savić; Novo Hopovo, 10.09.2013., on C. betulus, branch, F.G. 467, leg. $\&$ det. D. Savić.

Serbia: NP Đerdap and Karakuša, Karadžić 2011: 3, on $C$. betulus.

11. Sclerencoelia fascicularis (Alb. \& Schwein.) Pärtel \& Baral Fruška Gora: Beočin, 29.01.2013., on Populus alba and Fraxinus $s p$., branch, F.G. 263, leg. \& det. D. Savić.

Serbia: Sokobanja, Marković 2012: 44, on Prunus avium.

12.*Trochila craterium (DC.) Fr.

Fruška Gora: Brankovac, 22.03.2015., on Hedera helix, leaf, F.G. 573, leg. \& det. D. Savić. 
13.*Trochila laurocerasi (Desm.) Fr.

Fruška Gora: Iriški Venac, 10.03.2015., on Prunus laurocerasus, leaf, F.G. 264, leg. \& det. D. Savić.

14.*Velutarina rufo-olivacea (Alb. \& Schwein.) Korf Fruška Gora: Andrevlje, 28.02.2013., on Acer pseudoplatanus, branch, F.G. 193, leg. \& det. D. Savić.

Fam.: Chaetomellaceae Baral, P.R. Johnst. \& Rossman 2015

Chaetomella atra Fuckel (Anamorphic Zoellneria)

Serbia: Beograd (Topčider), Ranojević 1910: 386, on Melilotus albus.

Fam.: Chlorociboriaceae Baral \& P.R. Johnst. 2015

15. Chlorociboria aeruginascens (Nyl.) Kan. Ex Ram., Korf \& L.R. Batra

Fruška Gora: Andrevlje, Karaman et al. 2012: 44; Dumbovo, 09.10.2012., on Quercus sp., branch, F.G. 468, leg. \& det. D. Savić; Papratski Do, 27.10.2015., on Tilia tomentosa, branch, F.G. 335, leg. \& det. D. Savić.

Serbia: Mt Stara Planina, Ivančević 2007: 159.

16. Chlorociboria aeruginosa (Oeder) Seaver ex C.S. Ramamurthi, Korf \& L.R. Batra

Fruška Gora: Testera, 16.04.2015., on Quercus petraea, dead wood, F.G. 119, leg. \& det. D. Savić.

Serbia: Mt Tara (Crveni Potok), Čolić 1967: 411, sub Chlorosplenium aeruginosum.

Fam.: Cordieritidaceae Sacc. 1889

17.*Ionomidotis fulvotingens (Berk. \& M.A. Curtis) E.K. Cash

Fruška Gora: Erdelj, 31.03.2015., on Fraxinus ornus, dead wood, FG99, leg. \& det. D. Savić.

Fam.: Dermateaceae Fr. 1849

18.*Dermea cerasi (Pers.) Fr.

Fruška Gora: Iriški Venac, 16.04.2014., on Prunus avium, dead wood, F.G. 469, leg. \& det. D. Savić.

19.*Dermea prunastri (Pers.) Fr.

Fruška Gora: Erdelj, 31.03.2015., on Prunus spinosa, branch, F.G. 266, leg. \& det. D. Savić.

20. Pezicula carpinea (Pers.) Tul. ex Fuckel

Fruška Gora: Karadžić 2011: 4, on Carpinus betulus. 
21. Pezicula cinnamomea (DC.) Sacc.

Fruška Gora: Stražilovo, 15.10.2015., on Corylus avellana, branch, F.G. 411, leg. \& det. D. Savić.

Serbia: Karadžić 2006: 51, on Quercus petraea; northeastern part, Karadžić \& Milijašević 2005: 75, on Q. petraea.

22.*Pezicula ocellata (Pers.) Seaver

Fruška Gora: Sviloš, 14.03.2014., on Salix alba, branch, F.G. 194, leg. \& det. D. Savić.

Fam.: Drepanopezizaceae Bat. \& H. Maia 1960

Blumeriella jaapii (Rehm) Arx

Serbia: Mt Tara, Marković 2012: 23, on Prunus avium, leaf.

Diplocarpon alpestri (Ces.) Rossman

Serbia: Mt Kopaonik (Srebrnac), Ranojević 1914: 413, sub Bostrichonema alpestre, on Polygonum bistorta, anamorph.

Diplocarpon rosae F.A. Wolf

Serbia: Cannon 1996.

23. Drepanopeziza punctiformis Gremmen

Fruška Gora: Beočin, Karaman et al. 2012: 43, sub Marssonina brunnea, on Populus nigra, leaf, anamorph.

24. Drepanopeziza sphaerioides (Pers.) Nannf.

Fruška Gora: Beočin, Karaman et al. 2012: 43, sub Marssonina salicina, on Salix alba, leaf, anamorph.

Serbia: Marković \& Karadžić 2006: 186, sub Marssonina salicicola, on Salix sp., anamorph; Marković et al. 2007: 78, sub M. salicicola, on Salix spp., anamorph; Central Danube Region, Marković 2006: 72, sub M. salicicola, on Salix sp., anamorph.

Leptotrochila campanulae (DC.) Rossman

Serbia: Pirot (Sarlak), Ranojević 1910: 386, sub Placosphaeria campanulae, on Campanula bononiensis; Mt Avala, Ranojević 1914: 405, sub P. campanulae, on C. trachelium)

Marssonina andurnensis (Ces.) Magnus (Anamorphic Diplocarpon) Serbia: Beograd (Topčider), Ranojević 1910: 394, on Thymelaea passerina.

Marssonina truncatula (Sacc.) Magnus (Anamorphic Diplocarpon) Serbia: Poćuta, Ranojević 1910: 394, sub Marssonia truncatula, on Acer campestre, anamorph; Beograd (Topčider), Ranojević 1914: 412, sub M. truncatula, on Acer negundo, anamorph. 
10 Savić, D., Karaman, M.: LeOtiomycetes Checklist of Mt FruŠKa Gora

Pseudopeziza medicaginis (Lib.) Sacc.

Serbia: Vranjska Banja, Ranojević 1910: 353, sub Pseudopeziza trifolii f. medicaginis, on Medicago carstiensis; Avala, Ranojević 1914: 394, sub P.t. var. medicaginis, on Medicago lupulina.

Pseudopeziza trifolii (Biv.) Fuckel

Serbia: Ranojević 1914: 394, on Medicago sativa.

Fam.: Gelatinodiscaceae S.E. Carp. 1976

25. Ascocoryne cylichnium (Tul.) Korf

Fruška Gora: Dumbovo, Karaman et al. 2012: 43; Andrevlje, 15.10.2013., on Fagus sylvatica, dead wood, F.G. 120, leg. \& det. D. Savić.

Serbia: more locations, Karadžić 2003: 61, on Fagus sylvatica.

26. Ascocoryne sarcoides (Jacq.) J.V. Groves

Fruška Gora: Iriški Venac, Karaman et al. 2012: 43; Paragovo, Karaman et al. 2012: 43; Stražilovo, 16.10.2015., F.G. 470, leg. \& det. D. Savić.

Serbia: more locations, Karadžić 2003: 61, on F. sylvatica; Karadžić \& Milijašević 2003: 27, on $F$. sylvatica.

27.*Ascocoryne solitaria (Rehm) Dennis

Fruška Gora: Stražilovo, 16.10.2015., anamorph, F.G. 336, leg. \& det. D. Savić.

28.*Ascocotremella faginea (Peck) Seaver

Fruška Gora: Stražilovo, 06.11.2015., on Fagus sylvatica, dead wood, F.G. 412, leg. \& det. D. Savić.

Neobulgaria pura (Pers.) Petr.

Serbia: Karadžić \& Milijašević 2003: 28, on Fagus sylvatica; more locations, Karadžić 2003: 65, on F. sylvatica.

29.* Ombrophila violacea (Hedw.) Fr.

Fruška Gora: Popovica, 22.08.2013., on deciduous tree, dead wood, F.G. 471, leg. \& det. D. Savić; Rakovac, 06.06.2012., on Tilia tomentosa, dead wood, F.G. 195, leg. \& det. D. Savić; Stražilovo, 26.12.2016., on Fagus sylvatica, dead wood, F.G. 870, leg. \& det. D. Savić.

Fam.: Godroniaceae Baral 2015

Gremmeniella abietina (Lagerb.) M. Morelet

Serbia: Karadžić \& Stanivuković 2010: 4, on Pinus nigra and Pinus sylvestris; Kopaonik, Vlasina and Mt Goč, Karadžić \& Milanović 2008: 108, on P. nigra and P. sylvestris; more locations, Karadžić \& Milijašević 2008: 150, on P. nigra and $P$. syl- 
vestris; NP Kopaonik, Karadžić et al. 2002: 112, on P. nigra and P. sylvestris.

Fam.: Helotiaceae Rehm 1892

30. ${ }^{*}$ Cudoniella clavus (Alb. \& Schwein.) Dennis

Fruška Gora: Paragovo, 24.09.2013., on Carex silvatica, leaf, F.G. 337, leg. \& det. D. Savić.

31.*Cyathicula coronata (Bull.) Rehm

Fruška Gora: Paragovo, 29.10.2012., on herbaceous plant, dead stem, F.G. 267, leg. \& det. D. Savić; Grgurevci, 16.10.2015., on Clematis vitalba, dead stem, F.G. 121, leg. \& det. D. Savić.

32.*Cyathicula culmicola (Desm.) De Not.

Fruška Gora: Erdelj, 16.05.2014., on Dactylis glomerata, dead stem, F.G. 472, leg. \& det. D. Savić.

33.*Cyathicula cyathoidea (Bull.) Thüm.

Fruška Gora: Stražilovo, 15.10.2012., on herbaceous stem, F.G. 413, leg. \& det. D. Savić; Rajkovac, 20.02.2013., on Anthriscus sylvestris, dead stem, F.G. 548, leg. \& det. D. Savić; Rakovac, 07.05.2015., on Sambucus ebulus, dead stem, F.G. 547, leg. \& det. D. Savić.

34.*Hymenoscyphus aesculi (Velen.) Baral \& E. Rubio

Fruška Gora: Andrevlje, 09.09.2016., on Aesculus hippocastanum, petiole, F.G. 847, leg. \& det. D. Savić.

35. *Hymenoscyphus albidus (Gillet) W.Phillips

Fruška Gora: Banoštor, 11.10.2012., on Fraxinus excelsior, branch, F.G. 196, leg. \& det. D. Savić.

36. Hymenoscyphus calyculus (Sowerby) W. Phillips

Fruška Gora: Iriški Venac, Karaman et al. 2012: 43; Brankovac, 26.06.2013., on Fraxinus ornus, branch, leg. \& det. D. Savić.

37.*Hymenoscyphus caudatus (P.Karst.) Dennis

Fruška Gora: Susek, 18.09.2013., on Fraxinus excelsior, leaf, F.G. 474, leg. \& det. D. Savić.

38. Hymenoscyphus fructigenus (Bull.) Fr.

Fruška Gora: Ledinci, Karaman et al. 2012: 43; Iriški Venac, 26.10.2012., on Carpinus betulus, fruit, F.G. 574, leg. \& det. D. Savić; Bukovac, 12.08.2013., on Quercus petraea, cupula, F.G. 414, leg. \& det. D. Savić; Rakovac, 17.09.2014., on Fagus sylvatica, cupula, F.G. 338, leg. \& det. D. Savić.

39. *Hymenoscyphus humuli (Lasch) Dennis

Fruška Gora: Popovica, 06.10.2015., on Humulus lupulus, dead branch, F.G. 122, leg. \& det. D. Savić. 
12 Savić, D., Karaman, M.: LeOtiomycetes Checklist of Mt FruŠKa Gora

40.* Hymenoscyphus peruni (Velen.) Svrček

Fruška Gora: Paragovo, 12.12.2016., on Fagus sylvatica, dead branch, F.G. 848, leg. \& det. D. Savić.

41.*Hymenoscyphus scutula (Pers.) W.Phillips

Fruška Gora: Sremska Kamenica, 08.10.2012., on herbaceous plant, dead stem, leg. \& det. D. Savić; Paragovo, 19.09.2013., on herbaceous plant, dead stem, leg. \& det. D. Savić; Grgurevci, 16.10.2015., on Clematis vitalba, dead wood, F.G. 576, leg. \& det. D. Savić.

42.*Hymenoscyphus serotinus (Pers.) W.Phillips

Fruška Gora: Papratski Do, 21.11.2012., on Fagus sylvatica, branch, F.G. 268, leg. \& det. D. Savić; Vrdnik, 13.11.2014., on F. sylvatica, branch, F.G. 475, leg. \& det. D. Savić.

43.*Hymenoscyphus syringicolor Svrček

Fruška Gora: Iriški Venac, 13.12.2011., on Fagus sylvatica, leaf, F.G. 549, leg. \& det. D. Savić.

44.*Malotium paludosum Velen.

Fruška Gora: Iriški Venac, 28.04.2014., on Aconitum lycoctonum, leaf, F.G. 473, leg. \& det. D. Savić.

45. Phaeohelotium fagineum (Pers.) Hengstm.

Fruška Gora: Paragovo, 25.09.2013., on Fagus sylvatica, cupula, leg. \& det. D. Savić; Iriški Venac, 03.10.2013., on F. sylvatica, cupula, F.G. 577, leg. \& det. D. Savić.

Serbia: Karadžić \& Milijašević 2003: 28, sub Hymenoscyphus fagineus, on Fagus sylvatica; more locations, Karadžić 2003: 62, sub $H$. fagineus, on $F$. sylvatica L.; Tara (Crveni Potok), Čolić 1967: 411, sub Ombrophila faginea, on F. sylvatica.

46. ${ }^{*}$ Phaeohelotium geogenum (Cooke) Svrček \& Matheis

Fruška Gora: Vrdnik, 25.10.2013., on Quercus petraea, cupula, F.G. 197, leg. \& det. D. Savić.

47.*Phaeohelotium imberbe (Bull.:Fr.) Svrček

Fruška Gora: Andrevlje, 20.08.2013., on deciduous tree, branch, F.G. 476, leg. \& det. D. Savić.

48.* Phaeohelotium immutabile (Fuckel) Declercq

Fruška Gora: Popovica, 30.10.2016., on Quercus petraea, leaf, F.G. 849 , leg. \& det. D. Savić.

49.*Phaeohelotium monticola (Berk.) Dennis

Fruška Gora: Stražilovo, 18.08.2014., on Quercus petraea, cupula, seed, branch, Carpinus betulus, seed, F.G. 339, leg. \& det. D. Savić. 
Phaeohelotium umbilicatum (Le Gal) Dennis

Serbia: Bojčinska Šuma, Ivančević \& Davidović 2011: 25.

50. *Symphyosirinia clematidis Baral

Fruška Gora: Čerević, 18.03.2014., on Clematis vitalba, seed, anamorph, F.G. 269, leg. \& det. D. Savić.

Fam.: Heterosphaeriaceae Rehm 1888

Heteropatella lacera Fuckel (Anamorphic Heterosphaeria)

Serbia: Mt Stara Planina (Midžor), Ranojević 1910: 394, on Verbascum sp., anamorph)

51.*Heterosphaeria patella (Tode) Grev.

Fruška Gora: Banstol, 09.09.2013., on Apiaceae, dead stem, F.G. 123, leg. \& det. D. Savić.

Fam.: Hyaloscyphaceae Nannf. 1932

52.*Cistella aconiti (Rehm) Raitv. \& Järv

Fruška Gora: Iriški Venac, 28.04.2014., on Aconitum lycoctonum, dead stem, F.G. 198, leg. \& det. D. Savić; Ledinci, 27.11.2015., on Urtica dioica, dead stem, leg. \& det. D. Savić; Rakovac, 01.06.2016., on Arctium lappa, dead stem, leg. \& det. D. Savić.

53. Cistella acuum (Alb. \& Schwein.) Svrček

Fruška Gora: Iriški Venac, 03.06.2016., on Picea abies, leaves, F.G. 850 , leg. \& det. D. Savić.

Serbia: Karadžić \& Stanivuković 2010: 4, sub Dasyscyphus acuum, on Pinus sylvestris; more locations, Karadžić \& Milijašević 2008: 150, sub D. acuum, on $P$. sylvestris.

54. ${ }^{*}$ Cistella graminicola (Raitv.) Raitv.

Fruška Gora: Stražilovo, 10.06.2016., on Carex silvatica, leaves, F.G. 851, leg. \& det. D. Savić.

55.* Cistella grevillei (Berk.) Raitv.

Fruška Gora: Paragovo, 31.03.2014., on Equisetum telmateia, dead stem, F.G. 550, leg. \& det. D. Savić; Erdelj 07.05.2014., on Urtica dioica, dead stem, F.G. 415, leg. \& det. D. Savić.

56. *Fuscolachnum misellum (Roberge ex Desm.) J.H. Haines

Fruška Gora: Andrevlje, 27.10.2016., on Rubus hirtus, leaves, F.G. 852, leg. \& det. D. Savić.

57.*Hyaloscypha aureliella (Nyl.) Huhtinen

Fruška Gora: Iriški Venac, 03.03.2014., on Pinus nigra, dead wood, F.G. 270, leg. \& det. D. Savić. 
14 Savić, D., Karaman, M.: LeOtiomycetes Checklist of Mt FruŠKa Gora

58.*Hyaloscypha britannica Huhtinen

Fruška Gora: Paragovo, 04.112014., on Picea abies, dead wood, F.G. 551, leg. \& det. D. Savić.

59. Hyaloscypha fuckelii Nannf.

Fruška Gora: Testera, 05.092014., on Alnus glutinosa, dead wood, F.G. 552, leg. \& det. D. Savić.

60. *Hyaloscypha intacta Svrček

Fruška Gora: Papratski Do, 27.10.2015., on Fagus sylvatica, dead wood, F.G. 340, leg. \& det. D. Savić.

61.* Hyaloscypha minuta (Spooner \& Dennis) Baral

Fruška Gora: Erdelj, 29.12.2016., deciduous tree, branch, F.G. 869 , leg. \& det. D. Savić.

62. ${ }^{*}$ Hyaloscypha quercicola (Velen.) Huntinen

Fruška Gora: Stražilovo, 27.03.2016., on Quercus petraea, bark, F.G. 553, leg. \& det. D. Savić.

63.* Olla millepunctata (Lib.) Svrček

Fruška Gora: Paragovo, 04.04.2013., on Rubus silesiacus, branch, F.G. 341, leg. \& det. D. Savić.

64. ${ }^{*}$ Psilocistella conincola (Velen.) Svrček

Fruška Gora: Paragovo, 25.12.2012., on Pinus nigra, cone, F.G. 416, leg. \& det. D. Savić; Iriški Venac, 09.12.2016., on Pinus nigra, cone, F.G. 871, leg. \& det. D. Savić.

Fam.: Lachnaceae Raitv. 2004

65.*Albotricha acutipila (P.Karst.) Raitv.

Fruška Gora: Paragovo, 28.03.2014., on Dactylis glomerata, dead stem, F.G. 124, leg. \& det. D. Savić.

66.*Brunnipila palearum (Desm.) Baral

Fruška Gora: Susek, 21.03.2013., on Alopecurus pratensis, dead stem, F.G. 554, leg. \& det. D. Savić; Ledinci, 04.05.2016., on Poaceae, dead stem, F.G. 199, leg. \& det. D. Savić.

67. * Capitotricha bicolor (Bull.) Baral

Fruška Gora: Testera, 21.02.2014., on Quercus sp., branch, F.G. 271, leg. \& det. D. Savić.

68.*Dasyscyphella claviculata (Velen.) Baral \& Svrček

Fruška Gora: Iriški Venac, 07.10.2016., on Quercus petraea, leaf, F.G. 853, leg. \& det. D. Savić, Zmajevac, 20.10.2016., on $Q$. petraea, leaf, F.G. 854, leg. \& det. D. Savić. 
69. Dasyscyphella nivea (R. Hedw.) Raitv.

Fruška Gora: Paragovo, 21.02.2007., F.G. 478, leg. \& det. D. Savić; Andrevlje, 16.10.2013., F.G. 578, leg. \& det. D. Savić; Crveni čot, 22.03.2013., F.G. 479, leg. \& det. D. Savić.

Serbia: Karadžić 2006: 51, sub Dasyscyphus niveus, on Quercus petraea; northeastern part, Karadžić \& Milijašević 2005: 74, sub $D$. niveus, on $Q$. petraea.

70.*Incrucipulum ciliare (Schrad.) Baral

Fruška Gora: Kraljeva Stolica, 17.10.2016., on Quercus petraea, leaf, F.G. 855, leg. \& det. D. Savić.

Lachnellula calyciformis (Battarra) Dharne

Serbia: Mt Zlatar, Mt Kopaonik and NP Tara, Karadžić 2008: 85, on Abies alba.

71. ${ }^{*}$ Lachnellula resinaria (Cooke \& W. Phillips) Rehm

Fruška Gora: Stražilovo, 18.08.2016., on Picea abies, decorticated wood, F.G. 856, leg. \& det. D. Savić.

Lachnum capitatum (Peck) Svrček

Serbia: northeastern part, Karadžić \& Milijašević 2005: 74, sub Dasyscyphus capitatus, on Quercus petraea.

72.* Lachnum caricis (Desm.) Hohn.

Fruška Gora: Paragovo, 25.02.2013., on Carex sylvatica, leaf, F.G. 342 , leg. \& det. D. Savić.

73. * Lachnum controversum (Cooke.) Rehm

Fruška Gora: Beočin, 14.06.2013., on Phragmites australis, dead stem, F.G. 417, leg. \& det. D. Savić.

74. ${ }^{*}$ Lachnum fasciculare Velen.

Fruška Gora: Jabuka, 28.11.2012., on deciduous tree, dead wood, F.G. 481, leg. \& det. D. Savić.

Lachnum fuscescens (Pers.) P. Karst.

Serbia: Karadžić \& Milijašević 2003: 27, sub Dasyscyphus fuscescens, on Fagus sylvatica; more locations, Karadžić 2003: 62, sub D. fuscescens, on F. sylvatica.

75. ${ }^{*}$ Lachnum impudicum Baral

Fruška Gora: Testera, 14.03.2014., on deciduous tree, dead wood, F.G. 555, leg. \& det. D. Savić.

Lachnum patulum (Pers.) Rehm

Serbia: northeastern part, Karadžić \& Milijašević 2005: 74, sub Dasyscyphus patulus, on Quercus petraea. 
Lachnum pudibundum (Quél.) J. Schröt.

Serbia: Marković \& Karadžić 2006: 184, sub Dasyscyphus pudibundus, on Salix sp.; Marković et al. 2007: 77, sub D. pudibundus, on Salix spp.; Central Danube Region, Marković 2006: 71, sub D. pudibundus, on Salix sp.

76.* Lachnum pudicellum. (Quél.) J. Schröt

Fruška Gora: Ledinci, 09.05.2016., on Poaceae, dead stem, F.G. 272, leg. \& det. D. Savić.

Lachnum pulverulentum (Lib.) P. Karst.

Serbia: more locations, Karadžić \& Milijašević 2008: 150, sub Dasyscyphus pulverulentus, on Pinus nigra.

77. Lachnum virgineum (Batsch) P.Karst.

Fruška Gora: Paragovo, Karaman et al. 2012: 43; Crveni čot, Karaman et al. 2012: 43; Iriški Venac, 21.02.2014., on Pinus nigra, cone, F.G. 579, leg. \& det. D. Savić.

78. ${ }^{*}$ Lasiobelonium nidulum (J.C. Schmidt. \& Kunze) Spooner

Fruška Gora: Petrovaradin, 16.09.2013., on herbaceous plant, dead stems, leg. \& det. D. Savić; Iriški Venac, 28.04.2015., on Polygonatum odoratum, dead stem, F.G. 125, leg. \& det. D. Savić.

79. ${ }^{*}$ Lasiobelonium variegatum (Fuckel) Raitv.

Fruška Gora: Andrevlje, 28.02.2013., on Tilia tomentosa, dead wood, leg. \& det. D. Savić; Sremska Kamenica, 04.02.2014., on deciduous tree, branch, leg. \& det. D. Savić; Papratski Do, 06.11.2015., on T. tomentosa, dead wood, F.G. 343, leg. \& det. D. Savić; Testera, 10.03.2016., on Pteridium aquilinum, leaf, F.G. 872 , leg. \& det. D. Savić

80. Neodasyscypha cerina (Pers.) Spooner

Fruška Gora: Irig, 27.06.2014., on Rosa canina, branch, F.G. 580, leg. \& det. D. Savić.

Serbia: Mt Avala, Ranojević 1910: 352, sub Dasyscypha cerina, on Fagus sylvatica.

81. Proliferodiscus pulveraceus (Alb. \& Schwein.) Baral Fruška Gora: Andrevlje, 23.01.2013., on Fagus sylvatica, wood, F.G. 581, leg. \& det. D. Savić; Paragovo, 22.01.2013., on deciduous tree, branch, F.G. 482, leg. \& det. D. Savić.

82. Trichopeziza mollissima Fuckel

Fruška Gora: Andrevlje, 27.03.2013., on Apiaceae, dead stem, F.G. 556, leg. \& det. D. Savić; Iriški Venac, 29.04.2014., on Aconitum lycoctonum, dead stem, F.G. 418, leg. \& det. D. Savić. 


\section{Trichopeziza sulphurea Fuckel}

Fruška Gora: Banoštor, 10.10.2012., on Urtica dioica, dead stem, leg. \& det. D. Savić., Paragovo, 24.09.2013., on herbaceous plant, dead stem, F.G. 273, leg. \& det. D. Savić.

Fam.: Mitrulaceae Rchb. 1828

\section{Mitrula paludosa Fr.}

Serbia: Mt Tara (Crveni Potok), Čolić 1967: 411.

\section{Fam.: Mollisiaceae Rehm 1891}

84. Mollisia cinerea (Batsch.) P.Karst.

Fruška Gora: Paragovo, Karaman et al. 2012: 43, on deciduous tree, dead wood); Papratski Do, 16.06.2014.; on Carpinus betulus, dead wood, leg. \& det. D. Savić.

Serbia: Kragujevac (Košutnjak) and Beograd (Topčider), Ranojević 1910: 353.

85. Mollisia fusca (Pers.) P. Karst

Fruška Gora: Stražilovo, 27.08.2013., on deciduous tree, dead wood, F.G. 484, leg. \& det. D. Savić.

Serbia: Mt Avala, Ranojević 1910: 352, sub Tapesia fusca, on Corylus avellana.

86. Mollisia ligni (Desm.) P.Karst.

Fruška Gora: Iriški Venac, 06.05.2014., on Carpinus betulus, dead wood, F.G. 557, leg. \& det. D. Savić.

Serbia: Beograd (Topčider), Ranojević 1910: 353, sub Mollisia lignicola, on Quercus cerris.

87. Mollisia lividofusca (Fr.) Gillet

Fruška Gora: Paragovo, 21.12.2016., on Pinus nigra, cone, F.G. 857, leg. \& det. D. Savić.

Serbia: Beograd (Košutnjak), Ranojević 1910: 352, sub Tapesia lividofusca, on Carpinus betulus.

88.*Mollisia luctuosa Boud.

Fruška Gora: Glavica, 15.03.2016., on Carex flacca, leaf, base of stem, F.G. 483, leg. \& det. D. Savić.

89.*Mollisia melaleuca (Fr.) Sacc.

Fruška Gora: Andrevlje, 28.02.2013., on deciduous tree, dead wood, F.G. 419, leg. \& det. D. Savić.

90.*Mollisia nervicola (Desm.) Gillet

Fruška Gora: Sremska Kamenica, 14.05.2015., on Quercus robur, leaf, F.G. 345, leg. \& det. D. Savić. 
18 SAvić, D., Karaman, M.: LeOtiomycetes Checklist of Mt FruŠKa Gora

91.*Mollisia olivaceocinerea Svrček

Fruška Gora: Brankovac, 11.03.2016., on Betula pendula, branch, F.G. 126, leg. \& det. D. Savić; Andrevlje, 13.09.2016., on deciduous tree, branch, leg. \& det. D. Savić, Bukovac, 24.11. 2016., on Pinus nigra, dead wood, F.G. 873, leg. \& det. D. Savić

92.* Mollisia phalaridis (Lib. ex P. Karst.) Rehm

Fruška Gora: Sremska Kamenica, 18.04.2016., on Poaceae, leaf, F.G. 200, leg. \& det. D. Savić.

93.* Mollisia retincola (Rabenh.) P. Karst.

Fruška Gora: Rakovac, 30.04.2013., on Phragmites australis, dead stem, F.G. 485, leg. \& det. D. Savić.

94.* Mollisia revincta (P. Karst.) Rehm

Fruška Gora: Paragovo, 19.05.2016., on Rubus sp., branch, F.G. 274, leg. \& det. D. Savić.

95. Mollisia rosae (Pers.) P. Karst.

Fruška Gora: Brankovac, 28.05.2013., on Rosa canina, branch, F.G. 558, leg. \& det. D. Savić.

Fam.: Pezizellaceae Velen. 1934

96.*Allophylaria byssacea P. Karst.

Fruška Gora: Brankovac, 04.11.2015., on Arctium lappa, leaf, F.G. 559, leg. \& det. D. Savić.

97.*Allophylaria filicum (W. Phillips) Svrček

Fruška Gora: Vrdnik, 08.11.2016., on Dryopteris filix-mas, leaf, F.G. 840 , leg. \& det. D. Savić.

98.*Antinoa pulchella (Fuckel) Baral

Fruška Gora: Bešenovački prnjavor, 25.11.2016., on Pinus nigra, leaf, F.G. 845, leg. \& det. D. Savić.

99. Calycellina alniella (Nyl.) Baral

Fruška Gora: Paragovo, Karaman et al. 2012: 43, sub Pezizella alniella; Testera, 20.02.2014., on Alnus glutinosa, catkin, leg. \& det. D. Savić.

100.* Calycellina chlorinella (Ces.) Dennis

Fruška Gora: Andrevlje, 24.11.2015., on Urtica dioica, dead stem, F.G. 486, leg. \& det. D. Savić.

Calycellina fagina (Ant. Schmidt \& Arendh.) Baral

Serbia: more locations, Karadžić 2003: 62, sub Scutoscypha fagi, on Fagus sylvatica. 
101.*Calycellina populina (Fuckel) Hohn.

Fruška Gora: Jabuka, 28.11.2012., on Fagus sylvatica, leaf, F.G. 560, leg. \& det. D. Savić; Ledinci, 22.11.2013., on F. sylvatica, leaf, F.G. 420, leg. \& det. D. Savić.

Calycellina punctata (Fr.) Lowen \& Dumont

Serbia: northeastern part, Karadžić \& Milijašević 2005: 73, sub Calycellina punctiformis, on Quercus petraea.

102.*Calycellina rubescens (Mouton) Van Vooren

Fruška Gora: Iriški Venac, 07.10.2016., on Quercus petraea, leaf, F.G. 858, leg. \& det. D. Savić.

103. Calycina citrina (Hedw.) Gray

Fruška Gora: Bukovac, Dumbovo, Iriški Venac, Papratski Do and Stražilovo, Karaman et al. 2012: 44, sub Bisporella citrina.

Serbia: Beograd (Topčider) and Mt Stara Planina (Međa Planina), Ranojević 1910: 352, sub Helotium citrinum; Mt Stara Planina, Ivančević 2007: 159, sub Bisporella citrina.

104. *alycina claroflava (Grev.) Kuntze

Fruška Gora: Stražilovo, 10.06.2016., on deciduous tree, dead wood, F.G. 859, leg. \& det. D. Savić.

105. Calycina conorum (Rehm) Baral.

Fruška Gora: Iriški Venac, 21.02.2007., on Pinus nigra, cone, F.G. 275, leg. \& det. D. Savić.

Serbia: Karadžić \& Stanivuković 2010: 5, sub Pezizella chionea, on Pinus nigra and Pinus sylvestris; more locations, Karadžić \& Milijašević 2008: 151, sub $P$. chionea, on $P$. nigra and $P$. sylvestris.

106.* Calycina cruentata (P. Karst.) Kuntze

Fruška Gora: Vrdnik, 10.11.2016., on Carex silvatica, leaf, F.G. 842 , leg. \& det. D. Savić.

107.*Calycina gemmarum (Boud.) Baral

Fruška Gora: Stražilovo, 17.04.2013., on Populus nigra 'Italica', fallen buds, F.G. 346, leg. \& det. D. Savić.

108. ${ }^{*}$ Calycina herbarum (Pers.) Gray

Fruška Gora: Kraljeva stolica, 10.10.2013., on herbaceous plant, dead stem, F.G. 487, leg. \& det. D. Savić; Jabuka, 11.10.2013., on herbaceous plant, dead stem, F.G. 488, leg. \& det. D. Savić.

109.*Calycina italica (Sacc.) Baral

Fruška Gora: Ledinci, 01.11.2016., on Quercus petraea, branch, F.G. 860, leg. \& det. D. Savić; Paragovo, 18.12.2015., on $Q$, petraea, branch, F.G. 861, leg. \& det. D. Savić. 
Calycina phyllophila (Desm.) Baral

Serbia: Karadžić \& Milijašević 2003: 28, sub Hymenoscyphus phyllophilus, on Fagus sylvatica; more locations, Karadžić 2003: 62 , on F. sylvatica.

110.*Calycina scolochloae (De Not.) Baral

Fruška Gora: Sremska Kamenica, 30.03.2015., on Phragmites australis, dead stem, F.G. 582, leg. \& det. D. Savić.

111.*Calycina subcitrina (Velen.) Baral

Fruška Gora: Sremska Kamenica, 24.10.2016., on Acer platanoides, leaf, F.G. 862, leg. \& det. D. Savić.

112.*Calycina subtilis (Fr.) Baral

Fruška Gora: Iriški Venac, 21.11.2016., on Pinus nigra, leaf, F.G. 843, leg. \& det. D. Savić.

113.*Calycina vulgaris (Fr.) Baral

Fruška Gora: Beočin, 12.12.2013., on Salix alba, branch, F.G. 561, leg. \& det. D. Savić.

114.*Mollisina rubi (Rehm) Höhn.

Fruška Gora: Andrevlje, 27.10.2016., on Rubus sp., leaf, leg. \& det. D. Savić.

Pezizella fagi (Jaap) Matheis

Serbia: Karadžić \& Milijašević 2003: 28, on Fagus sylvatica; more locations, Karadžić 2003: 62, on F. sylvatica.

115.* Ciliolarina laetifica Huhtinen

Fruška Gora: Iriški Venac, 03.03.2016., on Pinus nigra, dead wood, F.G. 421, leg. \& det. D. Savić; Paragovo, 21.11.2016., on Pinus nigra, dead wood, leg. \& det. D. Savić

116.*Ciliolarina laricina (Raitv.) Svrček

Fruška Gora: Stražilovo, 27.12.2016., on Picea abies, dead wood, F.G. 863 , leg. \& det. D. Savić

117.*Psilachnum chrysostigmum (Fr.) Raitv.

Fruška Gora: Iriški Venac, 28.04.2014., on Asplenium scolopendrium, leaf, F.G. 276, leg. \& det. D. Savić; 29.04.2014., on Dryopteris filix-mas, leaf, F.G. 489, leg. \& det. D. Savić; Testera, 09.03.2016., on Pteridium aquilinum, leaf, F.G. 490, leg. \& det.

D. Savić.

118.* Psilachnum eburneum (Roberge) Baral

Fruška Gora: Glavica, 15.03.2016., on Poa pratensis, leaf, F.G. 562, leg. \& det. D. Savić. 
119.*Psilachnum lanceolatoparaphysatum (Rehm) Höhn.

Fruška Gora: Brankovac, 08.05.2015., on Helleborus odorus, leaf, F.G. 347, leg. \& det. D. Savić.

120.* Psilachnum rubicundum (Sacc. \& Speg.) Baral

Fruška Gora: Iriški Venac, 03.06.2016., on Helleborus odorus, leaf, F.G. 201, leg. \& det. D. Savić.

$121{ }^{*}$ Rodwayella citrinula (P. Karst.) Spooner

Fruška Gora: Grgurevci, 08.02.2016., on Poa pratensis, dead stem, F.G. 422, leg. \& det. D. Savić.

Fam.: Ploettnerulaceae Kirschst. 1924

Pirottaea gallica Sacc.

Serbia: Beograd (Topčider), Ranojević 1914: 394, on Helleborus odorus.

122.*Pirottaea imbricata Nannf.

Fruška Gora: Andrevlje, 22.04.2016., on Rubus sp., branch, F.G. 277, leg. \& det. D. Savić.

123.*Pirottaea lychnidis (Desm.) Chlebická

Fruška Gora: Beočin, 11.03.2016., on Silene alba, dead stem, F.G. 563, leg. \& det. D. Savić.

124.*Pirottaea senecionis (Cooke \& W. Phillips) Nannf.

Fruška Gora: Andrevlje, 15.05.2015., on Centaurea scabiosa, dead stem, F.G. 491, leg. \& det. D. Savić.

125.*Pirottaea veneta Sacc. \& Speg.

Fruška Gora: Brankovac, 23.04.2015., on Helleborus odorus, leaf, F.G. 202, leg. \& det. D. Savić.

126.*Pyrenopeziza atrata (Pers.) Fuckel

Fruška Gora: Andrevlje, 22.04.2016., on Apiaceae, dead stem, F.G. 278, leg. \& det. D. Savić; Paragovo, 28.08.2015., on Angelica sylvestris, dead stem, F.G. 583, leg. \& det. D. Savić.

127.*Pyrenopeziza carduorum Rehm

Fruška Gora: Brankovac, 01.04.2013., on Centaurea scabiosa, dead stem, F.G. 423, leg. \& det. D. Savić.

128.* Pyrenopeziza rubi (Fr.) Rehm

Fruška Gora: Andrevlje, 22.04.2016., on Rubus sp., branch, F.G. 493, leg. \& det. D. Savić; Ledinci, 06.05.2016., on Rubus sp., branch, F.G. 492, leg. \& det. D. Savić.

Pyrenopeziza homogynes J. Schröt.

Serbia: Mt Kopaonik (Jelak), Ranojević 1914: 394, sub Pseudopeziza homogynes, on Homogyne alpina. 
Pyrenopeziza petiolaris (Alb. \& Schwein.) Nannf.

Serbia: Beograd (Košutnjak), Ranojević 1910: 354, sub Trochila petiolaris, on Aesculus hippocastanum.

Fam.: Rutstroemiaceae Holst-Jensen, L.M. Kohn \& T. Schumach. 1997

129. Rutstroemia bolaris (Batsch.) Rehm

Fruška Gora: Karadžić 2011: 4, on Carpinus betulus; Paragovo, Karaman et al. 2012: 44; Andrevlje, 05.09.2014., on Quercus sp., branch, leg. \& det. D. Savić.

Serbia: NP Đerdap and Karakuša, Karadžić 2011: 4, on Carpinus betulus.

Rutstroemia bulgarioides (P. Karst.) P. Karst.

Serbia: Mt Zlatar, Mt Kopaonik and NP Tara, Karadžić 2008: 85, on Picea abies; more locations, Keča 2006: 130.

130.* Rutstroemia echinophila (Bull.) Höhn.

Fruška Gora: Ledinci, 13.09.2005., on Quercus sp., cupula, F.G. 274, leg. \& det. D. Savić; Glavica, 30.08.2013., on Quercus sp., cupula, leg. \& det. D. Savić; Andrevlje, 05.09.2013., on Quercus sp., cupula, F.G. 584, leg. \& det. D. Savić; Papratski Do, 06.10.2015., on Quercus sp., cupula, F.G. 585, leg. \& det. D. Savić.

131. Rutstroemia firma Pers.

Fruška Gora: Ledinci, Karaman et al. 2012: 44; Papratski Do, 18.10.2012., on deciduous tree, branch, FG264, leg. \& det. D. Savić; Papratski Do, 06.10.2015., on Quercus petraea, branch, leg. \& det. D. Savić; Stražilovo, 13.10.2015., on Q. petraea, branch, F.G. 348, leg. \& det. D. Savić.

Serbia: Bojčinska šuma, Ivančević \& Davidović 2011: 26.

132.* Rutstroemia fruticeti Rehm

Fruška Gora: Brankovac, 08.11.2014., on Rubus silesiacus, branch, F.G. 864, leg. \& det. D. Savić.

133. *Rutstroemia luteovirescens (Roberge ex Desm.) W.L. White Fruška Gora: Iriški Venac, 29.10.2012., on Acer platanoides, branch, F.G. 279, leg. \& det. D. Savić; Jabuka, 11.10.2013., on $A$. platanoides, branch, F.G. 494, leg. \& det. D. Savić; Andrevlje, 15.10.2013., on A. platanoides, branch, F.G. 349, leg. \& det. D. Savić.

134. Rutstroemia petiolorum (Roberge ex Desm.) W.L. White Fruška Gora: Popovica, 06.10.2015., on Fagus sylvatica, leaf, petiole, F.G. 203, leg. \& det. D. Savić. 
Sebia: Karadžić \& Milijašević 2003: 28, on F. sylvatica; Karadžić 2006: 49, on Quercus petraea; more locations, Karadžić 2003: 62, on $F$. sylvatica; northeastern part, Karadžić \& Milijašević 2005: 75, on $Q$. petraea.

135.*Rutstroemia sydowiana (Rehm) W.L. White

Fruška Gora: Glavica, 30.08.2013., on Quercus petraea, leaf, petiole, F.G. 127, leg. \& det. D. Savić.

136.*Torrendiella ciliata Boud.

Fruška Gora: Andevlje, 27.10.2016., on Rubus hirtus, leaf, F.G. 838 , leg. \& det. D. Savić.

Fam.: Sclerotiniaceae Whetzel 1945

137. Botryotinia fuckeliana (de Bary) Whetzel

Fruška Gora: Kraljeva stolica, 23.02.2007., anamorph, F.G. 495, leg. \& det. D. Savić.

Serbia: Karadžić \& Milijašević 2003: 27, sub Botrytis cinerea, on Fagus sylvatica; Karadžić \& Milijašević 2008: 150, sub $B$. cinerea, on Pinus nigra and Pinus sylvestris; Karadžić 2006: 49, sub B. cinerea Pers., on Quercus petraea, Q. frainetto and $Q$. cerris; Keča 2006: 130, sub $B$. cinerea; Karadžić 2012: 3, sub $B$. cinerea, on F. sylvatica; Karadžić \& Stanivuković 2010: 3, sub $B$. cinerea, on $P$. nigra and $P$. sylvestris; Beograd (Topčider), Ranojević 1910: 395, sub B. cinerea, on Vitis vinifera and Ranunculus ficaria; northeastern part, Karadžić \& Milijašević 2005: 73, sub B. cinerea, on Q. petraea; more locations, Karadžić 2003: 61, sub B. cinerea, on $F$. sylvatica; Novi Sad, Marković 2012: 25, sub B. cinerea.

Botrytis elliptica (Berk.) Cooke (Anamorphic Botryotinia)

Serbia: Bulaić et al. 2006: 96, on Lilium sp. anamorph.

138. ${ }^{*}$ Botrytis galanthina (Berk. \& Broome) Sacc. (Anamorphic Botryotinia)

Fruška Gora: Brankovac, 18.02.2014., on Galanthus nivalis, anamorph, F.G. 280, leg. \& det. D. Savić.

139.*Ciboria amentacea (Balb.) Fuckel

Fruška Gora: Testera, 05.03.2013., on Alnus glutinosa, rotting catkins, F.G. 567, leg. \& det. D. Savić; Beočin, 06.02.2013., on Corylus avellana, rotting catkins, F.G. 566, leg. \& det. D. Savić; Glavica, 08.02.2014., on C. avellana, rotting catkins, F.G. 565, leg. \& det. D. Savić.

140. Ciboria batschiana (Zopf) N.F. Buchw.

Fruška Gora: Stražilovo, 13.10.2015., on Quercus petraea, acorn, F.G. 496, leg. \& det. D. Savić. 
Serbia: Karadžić \& Milijašević 2005: 75, sub Stromatinia pseudotuberosa, on Quercus petraea; northeastern part, Karadžić \& Milijašević 2005: 73, on Q. petraea; Stara Planina, Ivančević 2007: 160.

141. * Ciboria conformata (P.Karst.) Svrček

Fruška Gora: Testera, 20.08.2013., on Alnus glutinosa, leaf, F.G. 497, leg. \& det. D. Savić.

142.*Ciboria coryli (Schellenb.) N.F. Buchw.

Fruška Gora: Grgurevci, 08.02.2015., on Corylus avellana, rotting catkins, F.G. 865, leg. \& det. D. Savić.

143. ${ }^{*}$ Ciborinia bresadolae (Rick) J.T. Palmer Fruška Gora: Testera, 19.03.2014., on Quercus sp., branch, F.G. 568, leg. \& det. D. Savić; Papratski Do, 06.10.2015., on Quercus petraea, acorn, FG271, F.G. 569, leg. \& det. D. Savić.

Ciborinia candolleana (Lev.) Whetzel

Serbia: Karadžić 2006: 49, on Quercus petraea, Q. frainetto and Q. cerris L.; northeastern part, Karadžić \& Milijašević 2005: 73, on $Q$. petraea.

144. Dumontinia tuberosa (Bull.) L.M. Kohn

Fruška Gora: Iriški Venac, Karaman et al. 2012: 44; Rakovac, 26.02.2014., on Anemone ranunculoides, rhizome, F.G. 350, leg. $\&$ det. D. Savić.

Monilinia fructicola (G. Winter) Honey

Serbia: Hrustić et al. 2015

145. Monilinia fructigena Honey

Fruška Gora: Paragovo, 01.09.2015., on Malus sylvestris, fruit, anamorph, F.G. 128, leg. \& det. D. Savić.

Serbia: Ranojević 1910: 395, sub Monilia fructigena, on $M$. sylvestris, Pirus communis, Prunus domestica, P. avium and $P$. cerasus, anamorph; Hrustić et al. 2015.

146.*Monilinia johnsonii (Ellis \& Everh.) Honey

Fruška Gora: Glavica, 15.03.2016., on Crataegus monogyna, fruit, F.G. 424, leg. \& det. D. Savić.

Monilinia laxa (Aderh. \& Ruhland) Honey

Serbia: Miletić \& Stević 2006: 24; Hrustić et al. 2015; Beograd, Ranojević 1910: 395, sub Monilia cinerea, on Prunus avium, anamorph.

Sclerotinia minor Jagger

Serbia: Halaši et al. 2008, on Lactuca sativa. 
147. Sclerotinia sclerotiorum (Lib.) de Bary

Fruška Gora: Brankovac, 20.09.2014., on Helianthus annuus, anamorph, leg. \& det. D. Savić; Petrovaradin, 04.04.2016., leg. \& det. D. Savić; Iriški Venac, 15.04.2016., F.G. 204, leg. \& det. D. Savić.

Serbia: Beograd, Ranojević 1910: 352, sub Sclerotinia libertiana; Pančevo, Pavlović et al. 2006: 34, on Echinacea angustifolia and Echinacea purpurea.

148.* Sclerotinia trifoliorum Erikss.

Fruška Gora: Novo Hopovo, 23.10.2013., on Trifolium repens, leaf, F.G. 281, leg. \& det. D. Savić.

Strossmayeria lineage Jaklitsch et al. 2016

149.*Durella atrocyanea (Fr.) Höhn.

Fruška Gora: Iriški Venac, 16.04.2014., on Carpinus betulus, dead wood, F.G. 499, leg. \& det. D. Savić.

150.*Durella commutata Fuckel

Fruška Gora: Osovlje, 18.08.2014., on Quercus petraea, wood, F.G. 498, leg. \& det. D. Savić.

Durella connivens (Fr.) Rehm

Serbia: Karadžić \& Milijašević 2003: 27, on Fagus sylvatica; more locations, Karadžić 2003: 62, on F. sylvatica.

151.*Strossmayeria basitricha (Sacc.) Dennis

Fruška Gora: Paragovo, 04.07.2014., on Quercus sp., branch, F.G. 570, leg. \& det. D. Savić.

\section{Incertae sedis}

152.* Leptodontidium trabinellum (P. Karst.) Baral, Platas \& R. Galán Fruška Gora: Rakovac, 16.07.2014., on Fagus sylvatica, dead wood, F.G. 351, leg. \& det. D. Savić.

Monostichella salicis (Westend.) Arx (Anamorphic Drepanopeziza) Serbia: Marković et al. 2007: 78, on Salix spp., anamorph; Central Danube Region, Marković \& Karadžić 2006: 186, on Salix sp. anamorph.

153.*Polydesmia pruinosa (Gerd. ex Berk. \& Broome) Boud.

Fruška Gora: Andrevlje, 25.12.2013., on Nemania serpens, F.G. 129 , leg. \& det. D. Savić.

Rhynchosporium secalis (Oudem.) Davis (Anamorphic Helotiales) Serbia: Čoja 1998: 25, anamorph. 
26 Savić, D., Karaman, M.: LeOtiomycetes Checklist of Mt FruŠKa Gora

Ordo: LeOTIALES Korf \& Lizoň 2001

Fam.: Geoglossaceae Corda 1842

* Geoglossum fallax E.J. Durand

Serbia: Mt Kukavica (Slatina village), 28.11.2016., F.G. 846, leg.

S. Jovanović, det. D. Savić.

154. * Trichoglossum hirsutum (Pers.) Boud.

Fruška Gora: Bukovac, 17.11.2014., grassland, F.G. 425, leg. \& det. D. Savić.

Fam.: Leotiaceae Corda 1842

155. Leotia lubrica (Scop.) Pers.

Fruška Gora: Paragovo, Karaman et al. 2012: 44; Petrovaradinski rit, Karaman et al. 2012: 44; Stražilovo, 30.09.2014., on soil, F.G. 205, leg. \& det. D. Savić.

Ordo: PHACIDIALES Höhn 1917

Fam.: Phacidiaceae Fr. 1849

156. Bulgaria inquinans (Pers.) Fr.

Fruška Gora: Glavica, Karaman et al. 2012: 43; Paragovo, Karaman et al. 2012: 43; Stražilovo, Karaman et al. 2012: 43; Vorovo, Karaman et al. 2012: 43.

Serbia: Karadžić \& Milijašević 2003: 27, on Fagus sylvatica; Karadžić 2006: 52, on Quercus petraea, Q. frainetto and $Q$. cerris; Karadžić 2012: 3, on F. sylvatica; more locations, Karadžić 2003: 65 , on $F$. sylvatica; northeastern part, Karadžić \& Milijašević 2005: 73, on Q. petraea; Mt Tara, Ranojević 1910: 354, sub Bulgaria polymorpha, on F. sylvatica; Mt Stara Planina, Ivančević 2007: 159; Bojčinska šuma, Ivančević \& Davidović 2011: 24.

Gremmenia infestans (P. Karst.) Crous

Serbia: Karadžić \& Stanivuković 2010: 5, sub Phacidium infestans, on Pinus nigra and P. sylvestris; more locations, Karadžić \& Milijašević 2008: 152, sub $P$ h. infestans, on $P$. nigra and $P$. sylvestris; Mt Golija (Crepuljnik-Kamenito brdo), Gajić 1989: 60, sub Ph. infestans.

Phacidium lacerum Fr.

Serbia: more locations, Karadžić \& Milijašević 2008: 152, on Pinus sylvestris.

157. Phacidium lauri (Sowerby) Crous \& D. Hawksw.

Fruška Gora: Fruška Gora, Marković 2012: 26, sub Ceuthospora lauri, on Prunus avium; Sremska Kamenica, 21.03.2015., on Ilex aquifolium, leaf, F.G. 426, leg. \& det. D. Savić. 
Fam.: Tympanidaceae Baral \& Quijada 2015

158.*Claussenomyces atrovirens (Pers.) Korf \& Abawi Fruška Gora: Iriški Venac, 28.02.2014., Pinus nigra, decorticated wood, F.G. 866, leg. \& det. D. Savić.

159. Holwaya mucida (Schulzer) Korf. \& Abawi

Fruška Gora: Papratski Do, Karaman et al. 2012: 43; Iriški Venac, Karaman et al. 2012: 43.

160.*Tympanis alnea (Pers.) Fr.

Fruška Gora: Testera, 28.02.2014., on Alnus glutinosa, bark, F.G. 282, leg. \& det. D. Savić.

\section{Tympanis hypopodia $\mathrm{Nyl}$.}

Serbia: Karadžić \& Stanivuković 2010: 6, on Pinus nigra and $P$. sylvestris; more locations, Karadžić \& Milijašević 2008: 153, on P. nigra and P. sylvestris.

161.*Tympanis prunicola J.W. Groves

Fruška Gora: Bukovac, 21.03.2014., on Prunus spinosa, kora, F.G. 352, leg. \& det. D. Savić.

Ordo: Rhytismatales M.E. Barr ex Minter 1986

Fam.: Cudoniaceae P.F. Cannon 2001

Cudonia circinans (Pers.) Fr.

Serbia: Ivančević 1995: 148; Mt Tara (Crveni Potok), Čolić 1967: 413.

Fam.: Marthamycetaceae Baral, Lantz, Hustad \& Minter 2015

162. Cyclaneusma minus (Butin) DiCosmo, Peredo \& Minter Fruška Gora: Stražilovo, 21.03.2014., on Pinus nigra, leaf, F.G. 130, leg. \& det. D. Savić.

Serbia: Karadžić \& Stanivuković 2010: 4, on Pinus sylvestris; more locations, Karadžić \& Milijašević 2008: 150, on P. sylvestris; Golija, Gajić 1989: 60, sub Naemacyclus minor.

163. Cyclaneusma niveum (Pers.) DiCosmo

Fruška Gora: Popovica, 14.03.2016., on Pinus nigra, leaf, F.G. 206, leg. \& det. D. Savić.

Serbia: Karadžić \& Stanivuković 2010: 4, on P. nigra; more locations, Karadžić \& Milijašević 2008: 150, on P. nigra; Mt Golija, Gajić 1989: 60, sub Naemacyclus niveus; Suvobor, Golubović-Ćurguz et al. 2013: 166, sub N. niveus, on P. nigra. 
Naemacyclus fimbriatus (Schwein.) DiCosmo, Peredo \& Minter Serbia: Karadžić \& Stanivuković 2010: 4, sub Lasiostictis fimbriata, on Pinus sylvestris; more locations, Karadžić \& Milijašević 2008: 151, sub L. fimbriata, on P. sylvestris.

164. Propolis farinosa (Pers.) Fr.

Fruška Gora: Crveni čot, Karaman et al. 2012: 44; Stražilovo, 08.07.2013., on deciduous tree, dead wood); Andrevlje, 18.04. 2004., on deciduous tree, dead wood, leg. \& det. D. Savić.

Serbia: Beograd (Topčider) and Kragujevac (Košutnjak), Ranojević 1910: 354, sub Propolis faginea, on Quercus cerris and Cornus mas.

Fam.: Rhytismataceae Chevall. 1826

165. Coccomyces dentatus (J.C. Schmidt)

Fruška Gora: Sremska Kamenica, Schulzer et al. 1866: 57, sub Phacidium dentatum.

Serbia: Karadžić 2006: 49, sub Leptothyrium quercinum, on Quercus petraea, Q. frainetto and Q. cerris; northeastern part, Karadžić \& Milijašević 2005: 74, sub L. quercinum, on Quercus petraea.

166. Colpoma quercinum (Pers.) Wallr.

Fruška Gora: Iriški Venac, 29.12.2016., Quercus petraea, branch, F.G. 874, leg. \& det. D. Savić.

Serbia: Karadžić 2006: 51, on Quercus petraea; northeastern part, Karadžić \& Milijašević 2005: 74, on Q. petraea.

167.*Hypoderma ilicinum De Not.

Fruška Gora: Grgurevci, 17.10.2016., on Quercus petraea, leaf, F.G. 867, leg. \& det. D. Savić.

168.*Hypoderma rubi (Pers.) DC.

Fruška Gora: Paragovo, 07.02.2014., on Rubus silesiacus, branch, F.G. 353, leg. \& det. D. Savić; Brankovac, 25.02.2014., on $R$. silesiacus, branch, leg. \& det. D. Savić.

Hypohelion scirpinum (DC.) P.R. Johnst.

Serbia: Kijevo, Ranojević 1910: 354, sub Hypoderma scirpinum, on Schoenoplectus lacustris.

Lirula macrospora (R. Hartig) Darker

Serbia: Mt Tara (Crveni Potok), Čolić 1967: 443, sub Lophodermium macrosporum; Golija, Gajić 1989: 60; Zlatar, Kopaonik and NP Tara, Karadžić 2008: 85, on Picea abies. 
Lirula nervisequa (DC.) Darker

Serbia: Mt Tara (Crveni Potok), Čolić 1967: 443, sub Lophodermium nervisequum; Golija, Gajić 1989: 60; Mt Zlatar, Mt Kopaonik and NP Tara, Karadžić 2008: 85, on Abies alba.

Lophodermella sulcigena (Link) Höhn.

Serbia: Karadžić \& Stanivuković 2010: 4, on Pinus sylvestris; more locations, Karadžić \& Milijašević 2008: 151, on P. sylvestris.

169. Lophodermium arundinaceum (Schrad.) Chevall.

Fruška Gora: Sremska Kamenica, 15.03.2014., on Phragmites australis, leaf, FG123, leg. \& det. D. Savić.

Serbia: Krepoljin (Gradac), Ranojević 1910: 354, sub L. arundinaceum var. piptatheri, on Piptatherum paradoxum.

170.* Lophodermium caricinum (Roberge ex Desm.) Duby

Fruška Gora: Zmajevac, 21.04.2015., on Carex pilosa, leaf, F.G. 500, leg. \& det. D. Savić.

Lophodermium conigenum (Brunaud) Hilitzer

Serbia: Karadžić \& Stanivuković 2010: 4, on Pinus nigra and $P$. sylvestris; more locations, Karadžić \& Milijašević 2008: 151, on $P$. nigra and $P$. sylvestris.

171. Lophodermium foliicola (Fr.) P.F. Cannon \& Minter Fruška Gora: Glavica, 19.04.2016., on Crataegus monogyna, leaf, leg. \& det. D. Savić; Erdelj, 20.04.2016., on Pyrus communis, leaf, F.G. 207, leg. \& det. D. Savić.

Serbia: Kragujevac (Košutnjak), Ranojević 1910: 354, sub $L$. hysterioides, on Pyrus communis.

172. * Lophodermium gramineum (Fr.) Chevall.

Fruška Gora: Erdelj, 30.05.2013., on Poaceae, leaf, F.G. 283, leg. \& det. D. Savić; Glavica, 26.03.2014., on Poaceae, leaf, F.G. 501, leg. \& det. D. Savić.

173. Lophodermium juniperinum (Fr.) De Not.

Fruška Gora: Iriški Venac, 19.03.2015., on Juniperus horizontalis, leaf, FG86, leg. \& det. D. Savić.

Serbia: Deliblatska Peščara sands, Milijašević 2003: 165, sub $L$. juniperi, on Juniperus virginiana.

Lophodermium petiolicola Fuckel

Serbia: Karadžić 2006: 49, on Quercus petraea L.; northeastern part, Karadžić \& Milijašević 2005: 75, on Quercus petraea L.

Lophodermium piceae (Fuckel) Höhn.

Serbia: Mt Zlatar, Mt Kopaonik and NP Tara, Karadžić 2008: 85, on Picea abies. 
30 Savić, D., Karaman, M.: LeOtiomycetes Checklist of Mt FruŠKa Gora

174. Lophodermium pinastri (Schrad.) Chevall.

Fruška Gora: Iriški Venac, 10.04.2014., on Pinus nigra, leaf, FG198, leg. \& det. D. Savić.

Serbia: Milijašević \& Karadžić 2004: 122, on P. nigra; Karadžić \& Stanivuković 2010: 4, on P. nigra and P. sylvestris; more locations, Karadžić \& Milijašević 2008: 151, on P. nigra and $P$. sylvestris; Golija, Gajić 1989: 59, on P. nigra and P. sylvestris; Suvobor, Golubović-Ćurguz et al. 2013: 166, on P. nigra.

Lophodermium pini-excelsae S. Ahmad

Serbia: Karadžić \& Stanivuković 2010: 4, on Pinus sylvestris; more locations, Karadžić \& Milijašević 2008: 151, on P. sylvestris.

Lophodermium seditiosum Minter, Staley \& Millar

Serbia: Karadžić \& Stanivuković 2010: 4, on Pinus nigra and Pinus sylvestris; more locations, Karadžić \& Milijašević 2008: 151, on P. nigra and P. sylvestris; Golija, Gajić 1989: 59, on $P$. nigra and P. sylvestris; Suvobor, Golubović-Ćurguz et al. 2013: 166, on P. nigra.

Pseudorhytisma bistortae (DC.) Juel

Serbia: Mt Kopaonik (Srebrnac) and Suvo rudište, Ranojević 1914: 394, sub Pseudopeziza bistortae, on Persicaria bistorta.

175. Rhytisma acerinum Pers.

Fruška Gora: Iriški Venac, Ranković 1955., on Acer pseudoplatanus; Karaman et al. 2012: 44; Paragovo, 21.09.2014., on Acer campestre, leaf, F.G. 588, leg. \& det. D. Savić; Beočin, 29.09. 2013., on A. campestre, leaf, anamorph, F.G. 587, leg. \& det. D. Savić.

Serbia: Beograd (Topčider) and Mt Cer, Ranojević 1910: 354, on Acer campestre; Mt Suva Planina (Ravno Bučje), Ranojević 1910: 354, on Acer pseudoplatanus; Beograd (Topčider), Poćuta and Mt Suva Planina (Ravno Bučje), Ranojević 1910: 392, sub Melasmia acerina, on A. campestre and A. pseudoplatanus; Mt Tara (Crveni Potok), Čolić 1967: 443, on A. pseudoplatanus and A. platanoides.

176.*Rhytisma punctatum (Pers.) Fr.

Fruška Gora: Osovlje, 15.08.2014., on Acer tataricum, leaf, F.G. 354, anamorph, leg. \& det. D. Savić.

Rhytisma salicinum (Pers.) Fr.

Serbia: Mt Tara (Crveni Potok), Čolić 1967: 443, on Salix spp. 
Rhytisma umbonatum Hoppe

Serbia: Mt Tara (Crveni Potok), Čolić 1967: 443, sub Rhytisma amphigenum.

Therrya pini (Alb. \& Schwein.) Höhn.

Serbia: Karadžić \& Stanivuković 2010: 6, on Pinus sylvestris; more locations, Karadžić \& Milijašević 2008: 153, on P. sylvestris.

\section{Incertae sedis}

177.* Karstenia rhopaloides (Sacc.) Baral

Fruška Gora: Brankovac, 11.03.2016., on Salix caprea, bark, F.G. 284, leg. \& det. D. Savić.

Ordo: TheleBOlales P.F. Cannon 2001

Fam.: Thelebolaceae Eckblad 1968

178.*Thelebolus stercoreus Tode

Fruška Gora: Grgurevci, 02.12.2016., Oryctolagus cuniculus, dung, F.G. 868, leg. \& det. D. Savić.

179.*Trichobolus sphaerosporus Kimbr.

Fruška Gora: Andrevlje, 29.01.2013., on Capreolus capreolus, dung, F.G. 208, leg. \& det. D. Savić.

180.*Trichobolus zukalii (Heimerl) Kimbr.

Fruška Gora: Andrevlje, 29.01.2013., on Capreolus capreolus, dung, F.G. 209, leg. \& det. D. Savić.

Ordo: THELOCARPALES Lücking \& Lumbsch 2016

Fam.: Thelocarpaceae Zukal 1893

Thelocarpon laureri (Flot.) Nyl.

Serbia: Szabados 1998: 60.

\section{CONCLUSIONS}

In the period from 2012 to 2016 we investigated diversity of fungi from the class Leotiomycetes (Ascomycota) on Fruška Gora Mountain. In total we recorded 164 species of which 131 represent the first records for Serbia. For all presented species data of localities, collection dates, host plants and collectors was given, as well as data from the literature concerning all species that have so far been reported from Serbia (excluding Erysiphales). The total number of fungi from the class Leotiomycetes so far recorded in Serbia currently amounts to 342 (including 104 species from the order Erysiphales). 


\section{Acknowledgements}

The authors thank to Hans-Otto Baral for helpful advice and assistance in the identification of certain species, as well as to Selena Jovanovic for providing sample of Geoglossum fallax from Southern Serbia. This study was partially carried out within the project supported by the Ministry of Education, Science and Technological Development of the Republic of Serbia (III43002).

\section{REFERENCES}

Baral, H-O. (1987): Lugol`s solution/IKI versus Melzer`s reagent. - Mycotaxon 29: $399-450$.

Baral, H-O. (1992): Vital versus herbarium taxonomy: morphological differences between living and dead cells of ascomycetes, and their taxonomic implications. - Mycotaxon 44: 333-390.

Baral, H-O., Haelewaters, D., Pärtel, K. (2015) A new attempt to classify the families of the Helotiales. In: Anonimous: Second International Workshop on Ascomycete Systematics, 22-24 April 2015, Amsterdam. Programme: 26. Royal Netherlands Academy of Arts and Sciences, Amsterdam. (Abstract)

Bulaić, A., Đekić, I., Dukić, N., Duduk, B., Krstić, B. (2006) Alternaria dianthi prouzrokovač pegavosti i sušenja karanfila. In: Vukša, P. (ed.): VIII Savetovanje o zaštiti bilja, zbornik rezimea, Zlatibor, 27. novembar - 1. decembar 2006. godine: 95-96. - Društvo za zaštitu bilja Srbije, Beograd. (Abstract) [In Serbian]

Cannon, P.F. (1996) Systematics and diversity of the Phyllachoraceae associated with Rosaceae, with a monograph of Polystigma. - Mycological Research 100(12): 1409-1427.

Crous, P.W., Quaedvlieg, W., Hansen, K., Hawksworth, D.L., Groenewald, J.Z. (2014): Phacidium and Ceuthospora (Phacidiaceae) are congeneric: taxonomic and nomenclatural implications. - IMA Fungus 5(2): 173-193.

Čoja, M. (1998): A study of the barley parasite Rhynchosporium secalis in Serbia and development of sources of resistance. - Review of Research Work at the Faculty of Agriculture 43: 25-40.

Čolić, D. (1967): Sinekološka analiza flore gljiva u rezervatu sa pančićevom omorikom na Mitrovcu (Planina Tara). - Zaštita prirode 34: 389-505. [In Serbian]

Dennis, R.W.G. (1978): British Ascomycetes. - J. Cramer, Vaduz.

Ellis, M.B., Ellis, J.P. (1997): Microfungi on land plants an identification handbook. - The Richmond Publishing Co Ltd., UK.

Gajić, M. (1989): Flora i vegetacija Golije i Javora. - Šumarski fakultet, Beograd, OOUR Šumarstvo „Golija“, Ivanjica. [In Serbian] 
Gernandt, D.S., Platt, J.L., Spatafora, J.W., Holst-Jensen, A., Hamelin, R.C., Kohn, L.M. (2001): Phylogenetics of Helotiales and Rhytismatales based on partial small subunit nuclear ribosomal DNA sequences. - Mycologia 93: 915-933.

Golubović-Ćurguz, V., Karadžić, D., Gvozdić, Đ., Milenković, I., Radoičić-Antić, S. (2013): Success of pathogenic fungus Mycosphaerella pini Rost. and Munk. Control in austrian pine plantations on Suvobor. - Sumarstvo 2013(3-4): 163174. [In Serbian with English abstract]

Halaši, T.J., Halaši, R.J., Pajkert, A.A., Sokolova-Đokić, Lj.R. (2008): Fungal diseases of some vegetables grown in greenhouse and gardens. - Zbornik Matice srpske za prirodne nauke 114: 123-134.

Hrustić, J., Delibašić, G., Stanković, I., Grahovac, M., Krstić, B., Bulajić, A., Tanović, B. (2015): Monilinia Species Causing Brown Rot of Stone Fruit in Serbia. - Plant disease 99: 709-717.

Index Fungorum (2016): Authors of Fungal Names [http://www.indexfungorum. org/Names/AuthorsOfFungalNames.asp] (Accessed 1st march 2016)

Ivančević, B. (1995): Diverzitet makromiceta u Jugoslaviji sa pregledom vrsta od međunarodnog značaja. In: Stevanović, V., Vasić, V. (eds): Biodiverzitet Jugoslavije sa pregledom vrsta od međunarodnog značaja: 141-150. - Ecolibri, Biološki fakultet, Beograd. [In Serbian Latin]

Ivančević, B. (2007): Diverzitet makromiceta Stare planine. In: Lakušić, D., Ćetković, A. (eds): Biodiverzitet Stare planine u Srbiji. Rezultati projekta: „Prekogranična saradnja kroz upravljanje zajedničkim prirodnim resursima Promocija umrežavanja i saradnje između zemalja Jugoistočne Evrope“. Regionalni centar za životnu sredinu za centralnu i istočnu Evropu (REC) Kancelarija u Srbiji, Beograd. [In Serbian Latin]

Ivančević, B., Davidović, M. (2011): Makromicete na području Bojčinske šume i okvir za njihovo očuvanje. - Zaštita prirode 61(2): 21-33. [In Serbian with English abstract]

Janković, M.M., Mišić, V. (1980): Šumska vegetacija i fitocenoze Fruške gore. Matica srpska, Monografije Fruške gore, Novi Sad. [In Serbian]

Jaklitsch, W,M., Baralm H-O., Lücking, R., Lumbsch, H.T. (2016): Ascomycota. In: Frey, W. (ed.): Syllabus of Plant Families. Adolf Engler's Syllabus der Pflanzenfamilien 1/2. (ed. 13). - Borntraeger Science Publishers, Stuttgart.

Karadžić, D. (2003): The most important diseases in beech forests of Serbia. Šumarstvo 2003(1-2): 59-72. [In Serbian with English abstract]

Karadžić, D. (2006): Uticaj parazitskih gljiva na zdravstveno stanje stabala kitnjaka, sladuna i cera u prirodnim šumama i urbanim sredinama. - Šumarstvo 2006(3): 47-59. [In Serbian with English abstract]

Karadžić, D. (2008): The most frequent fungi disease agents in natural spruce and fir stands. - Šumarstvo 2008(3): 83-89. [In Serbian with English abstract]

Karadžić, D. (2011): The most important parasitic and saprophytic fungi on european hornbeam (Carpinus betulus L.) in Serbia and their role in tree decline. - Šumarstvo 2011(1-2): 1-11. [In Serbian with English abstract]

Karadžić, D. (2012): Uloga i značaj patogenih gljiva u sušenju bukve u Srbiji. Šumarstvo 2012(1-2): 1-16. [In Serbian with English abstract] 
Karadžić, D., Anđelić, M., Vemić, M. (2002): Gremmeniella abietina (Lagerb.) Morelet: distribution in Yugoslavia, significance and control. - Glasnik Sumarskog fakulteta Univerziteta u Beogradu 86: 111-122. [In Serbian with English abstract]

Karadžić, D., Lazarov, V., Milenković, M. (2004): Najznačajnije parazitske i saprofitske gljive na beloj imeli (Viscum album) i mogućnost njihovog korišćenja u biokontroli. - Glasnik šumarskog fakulteta Univerziteta u Beogradu 68: 115-126. [In Serbian with English abstract]

Karadžić, D., Milanović, S. (2008): Gremmeniella abietina (Lagerb.) Morelet: Distribution in Serbia and Montenegro, significance and control. - Glasnik šumarskog fakulteta Univerziteta u Beogradu 98: 107-116.

Karadžić, D., Milijašević, T. (2003): The most frequent parasitic and saprophytic fungi in coppice beech forests in Serbia. - Šumarstvo 2003(1-2): 25-35. [In Serbian with English abstract]

Karadžić, D., Milijašević, T. (2005): The most frequent parasitic and saprophytic fungi on sessile oak in Serbia and their part in tree dying. - Šumarstvo 2005(3): 71-84. [In Serbian with English abstract]

Karadžić, D., Milijašević, T. (2008): The most important parasitic and saprophytic fungi in austrian pine and scots pine plantations in Serbia. - Bulletin of the Faculty of Forestry 97: 147-170.

Karadžić, D., Stanivuković, N. (2010): The most important phytopatological problems in austrian pine and scots pine plantations in Serbia and the Republic Srpska. - Šumarstvo 2010(3-4): 1-4. [In Serbian with English abstract]

Karaman, M., Novaković, M., Savić, D., Matavulj, M. (2012): Preliminary checklist of Myxomycota and Ascomycotas from Fruška Gora Mountain. Zbornik Matice srpske za prirodne nauke 123: 37-49.

Keča, N. (2006): Prilog proučavanju mikoflore semena šumskog drveća. In: Vukša, P. (ed.): VIII Savetovanje o zaštiti bilja, zbornik rezimea, Zlatibor, 27. novembar - 1. decembar 2006. godine: 129-130. - Društvo za zaštitu bilja Srbije, Beograd. (Abstract) [In Serbian]

Kimbrough, J.W. (1970): Current trends in the classification of Discomycetes. Botanical Review 36: 91-161.

Kirk, P.M., Cannon, P.F., Minter, D.W., Stalpers, J.A. (2008): Dictionary of the Fungi (ed. 10). - CABI, Wallingford, UK.

Lumbsch, H.T., Huhndorf, S.M. (2011): Myconet volume 14. Part one. Outline of Ascomycota 2009. Part Two. Notes on Ascomycete Systematics. Nos. 47515113. - Fieldiana Life and Earth Sciences 1: 1-64.

Marković, M. (2006): Fungi agents of disease on willow bark. - Šumarstvo 2006(4): 69-79. [In Serbian with English abstract]

Marković, M. (2012): Investigation of parasitic funghi on wild cherry (Prunus avium L.) with special accent on bioecology of Daedaleopsis confragosa (Bilt.:Fr.) J.Schroet. - University of Belgrade, Faculty of Forestry, Belgrade. (PhD Thesis, manuscr.) [In Serbian with English abstract]

Marković, M., Karadžić, D. (2006): The most significant parasitic and saprophytic fungi on willows in the area of Central Danube basin. - Glasnik sumarskog 
fakulteta Univerziteta u Beogradu 94: 181-196. [In Serbian with English abstract]

Marković, M., Karadžić, D., Lazarev, V., Pap, P., Poljaković-Pajnik, L. (2007): Mycological Complex on the Leaves and Bark of Salix Species in Central Danube Basin. In: Szabó, I. (ed.): Proceedings of the Conference of IUFRO Working Party 7.02.02 21- 26 May 2007, Sopron, Hungary. Foliage, Shoot and Stem Diseases of Forest Trees. - Acta Silvatica \& Lignaria Hungarica Special Edition 2007: 75-80.

Medardi, G. (2012): Atlante fotografico degli Ascomiceti d'Italia. A.M.B. Centro Studi Micologici.

Milijašević, T. (2003): Najčešće parazitske i saprofitske gljive na nekim vrstama iz familije Cupressaceae. - Glasnik Šumarskog fakulteta Univerziteta u Beogradu 87: 161-173. [In Serbian with English abstract]

Milijašević, T., Karadžić, D. (2004): Parasitic and saprohytic fungi occuring in connexion with Sphaeropsis sapinea Dyko \& Sutton. - Glasnik Šumarskog fakulteta Univerziteta u Beogradu 90: 119-128. [In Serbian with English abstract]

Miletić, N., Stević, M. (2006): Problem Taphrina deformans i Monillinia spp. na koštićavom voću sa posebnim osvrtom na 2006. godinu. In: Vukša, P. (ed.): VIII Savetovanje o zaštiti bilja, zbornik rezimea, Zlatibor, 27. novembar - 1. decembar 2006. godine: 24-25. - Društvo za zaštitu bilja Srbije, Beograd. (Abstract) [In Serbian]

Miljković, N. (1975): Zemljišta Fruške gore. - Monografije Fruške gore I, Matica srpska, Novi Sad.

Pavlović, S., Stojšin, V., Jasnić, S., Stojanović, S. (2006): Prvi nalaz Sclerotinia sclerotiorum na Echinacea angustifolia i E.purpurea u Srbiji. In: Vukša, P. (ed.): VIII Savetovanje o zaštiti bilja, zbornik rezimea, Zlatibor, 27. novembar - 1. decembar 2006. godine: 34-35. - Društvo za zaštitu bilja Srbije, Beograd. (Abstract) [In Serbian]

Pfister, D.H., Kimbrough, J.W. (2001): Discomycetes. In: McLaughlin, D.J., McLaughlin, E.G., Lemke, P.A. (eds): The Mycota VII Part A. Systematics and Evolution: 257-281. - Berlin \& Heidelberg Springer-Verlag.

Ranković, B. (1955): Prilog poznavanju gljiva naših rezervata - (gljive Fruške gore). - Zavod za zaštitu prirode i naučno proučavanje prirodnih retkosti Narodne Republike Srbije, Naučna knjiga, Beograd. [In Serbian]

Ranković, B. (2002): Fungi from the genus Erysiphales and their parasites in Serbia. - Prirodno-matematički fakultet, Kragujevac.

Ranojević, N. (1910): Zweiter Beitrag zur Pilzflora Serbiens. - Annales Mycologici 8(3): 347-402.

Ranojević, N. (1914): Dritter Beitrag zur Pilzflora Serbiens. - Annales Mycologici 12(4): 393-421.

Schulzer, S., Kanitz, A., Knapp, J.A. (1866): Die bisher bekannten Pflanzen Slavoniens, ein Versuch. - Verhandlungen der Zoologisch-Botanischen Gesellschaft Wien 16: 3-172. 
Stevanović, V. (1995): Biogeografska podela teritorije Jugoslavije. In: Stevanović, V. \& Vasić, V. (eds): Biodiverzitet Jugoslavije, sa pregledom vrsta od međunarodnog značaja: 117-127. - Ecolibri, Biološki fakultet, Beograd. [In Serbian Latin]

Stevanović, V., Stevanović, B. (1995): Osnovni klimatski, geološki i pedološki činioci biodiverziteta kopnenih ekosistema Jugoslavije. In: Stevanović, V., Vasić, V. (eds): Biodiverzitet Jugoslavije, sa pregledom vrsta od međunarodnog značaja: 75-95. - Ecolibri, Biološki fakultet, Beograd. [In Serbian Latin]

Szabados, K. (1998): Epiphytis lichens - indicators of undistrubed development of forest. - Ekologija 33(1-2): 57-63.

Wang, Z., Binder, M., Schoch, C.L., Johnston, P.R., Spatafora, J.W., Hibbett, D.S. (2006a): Evolution of helotialean fungi (Leotiomycetes, Pezizomycotina): a nuclear rDNA phylogeny. - Molecular Phylogenetics and Evolution 41: 295-312.

Wang, Z., Johnston, P.R., Takamatsu, S., Spatafora, J.W., Hibbett, D.S. (2006b): Toward a phylogenetic classification of the Leotiomycetes based on rDNA data. - Mycologia 98(6): 1065-1075.

\title{
ГЉИВЕ КЛАСЕ LЕОТІОМУСЕТЕS НА ФРУШКОЈ ГОРИ СА ПРЕГЛЕДОМ НОВИХ ВРСТА ЗА СРБИЈУ
}

\author{
ДРАГИША САВИЋ, МАЈА КАРАМАН
}

\section{Р Е 3 И М Е}

У периоду од 2012. до 2016. године истраживан је диверзитет гљива класе Leotiomycetes (Ascomycota) на планини Фрушка гора. Укупно смо забележили 164 врста од којих 131 представља прве налазе за Србију. У раду је дата листа свих сакупљених врста припадника класе Leotiomycetes, осим реда Erysiphales, на Фрушкој гори, са подацима о локалитетима, датумима сакупљања и биљкама домаћинима, као и подаци из литературе о свим осталим врстама које су до сада нађене у Србији. Укупан број до сада евидентираних врста из класе Leotiomycetes у Србији износи 342, укључујући и врсте из реда Erysiphales (104). 
